\title{
Non-equilibrium transport properties of metal gated CNT-FET
}

\author{
Khurshed A. Shah*, M. Shunaid Parvaiz and Feroz A. Najar \\ Nanomaterials Research Laboratory, Department of Physics, \\ Govt. Degree College for Women, Anantnag, J\&K-192101-India \\ *Email: drkhursheda@gmail.com
}

Carbon Nanotubes finds various applications in nanoelectronics [1-4]. The current-voltage (IV) characteristics are one of the most important non-equilibrium transport properties of any device. Since the calculation of nonequilibrium transport properties of a device is a time-consuming work, therefore in this study we set the bias voltage in the range of 0 to $+2 \mathrm{~V}$. The IV-characteristic of the device has been obtained by using Huckel device (ATK-SE) calculator of the Atomistic Tool Kit Virtual Nanolab version 13.8.1 [5]. The I-V and conductance curves of the chosen top-gated CNT-FET structure along with transmission spectrum is shown in Figure 1 and Figure 2 at gate voltage of $0.5 \mathrm{~V}$ and $1 . \mathrm{V}$ respectively.

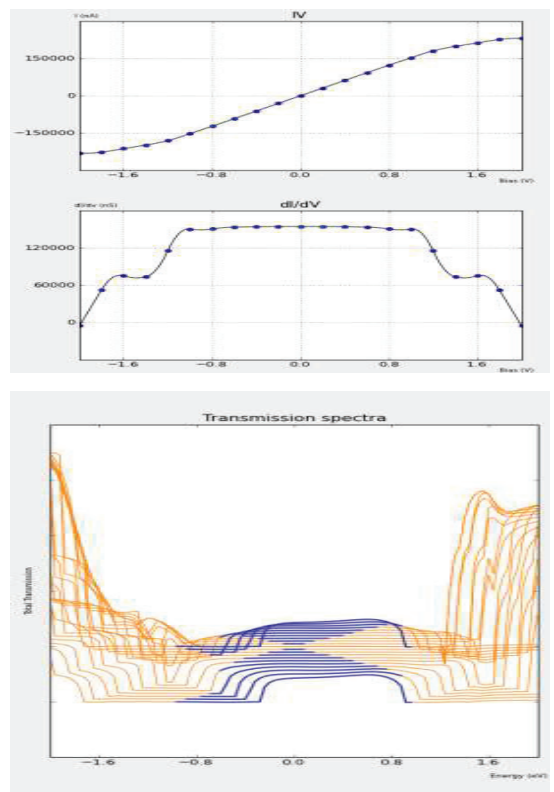

Figure 1: I-V and conductance curves of CNTFET at gate voltage of $0.5 \mathrm{~V}$

For every increase in gate voltage the current gets strengthened shows the behaviour of schottky diode effect. It is observed that the resistance of the channel (CNT) decreases with the increase in the gate voltage and thereby increases the current across the device. The conductance of the device remains constant upto $(\mathrm{V} \leq 0.8)$. However, it decreases sharply with further increases in the applied voltage. Besides this, the effect is more predominant when the gate voltage is increased from $0.5 \mathrm{~V}$ to $1.0 \mathrm{~V}$. The results are very helpful for designing newer CNT based devices [6].

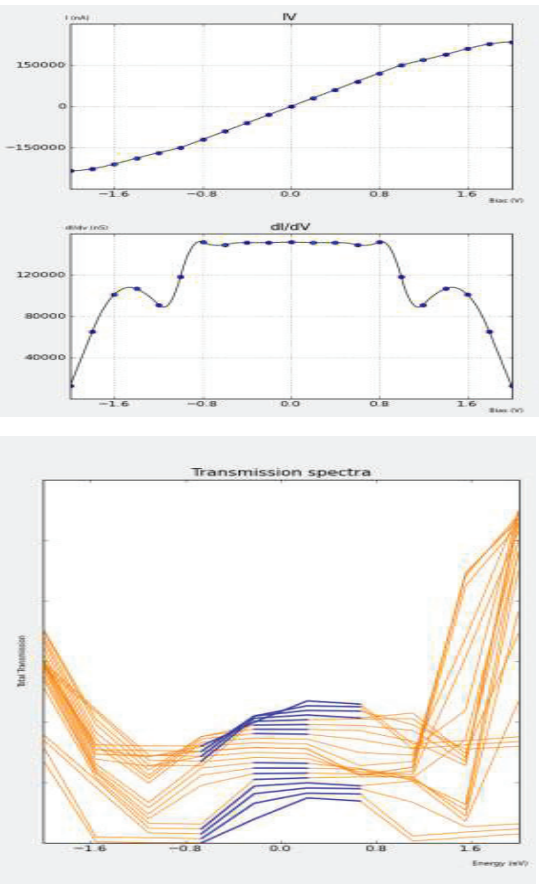

Figure 2: I-V and conductance curves of CNTFET at gate voltage of $0.1 \mathrm{~V}$.

\section{References}

1. W. Ren, J. Wang, Phys. Rev B. 69 (2004) 33306. 2. J. M. Soler, E. Artacho, J. D. Gale, A. Gracia, J. Junquera, P. Ordejon, D. Sanchz-Portal, Jour. Phys. 14 (2002) 2745.

3. J. P. Perdew, A. Zunger, Phys. Rev B. 23 (1981) 5048.

4. J. Taylor, H. Guo, J. Wang, Phys. Rev B. 63 (2001) 245407.

5. Atomistix ToolKit version 13.8.1, Quantumwise A/S (www.quantumwise.com).

6. S. Tiwari, Int. Jour. of Eng. Dev. and Res. 2 (2014) 3377. 